\title{
Associação Entre o Uso de Dispositivos Invasivos e Prolongamento do Tempo de Internação em uma Unidade de Terapia Intensiva Pediátrica
}

\author{
Association Between the Use of Invasive Devices and Prolongation of Hospital Stay \\ in a Pediatric Intensive Care Unit
}

\author{
Maria Mayara Araújo Pereira ${ }^{1}$ \\ Thais de Almeida da Silva ${ }^{2}$ \\ Ana Caroline Escarião de Oliveira ${ }^{3}$ \\ Juliana Almeida Marques Lubenow ${ }^{4}$
}

\section{RESUMO}

Objetivo: Averiguar se o uso de dispositivos invasivos leva ao prolongamento do tempo de internação na Unidade de Terapia Intensiva Pediátrica de um Hospital Universitário. Metodologia: Tratou-se de um estudo quantitativo do tipo descritivo, transversal e retrospectivo, realizado em um Hospital Universitário, onde foram usados dados do livro de admissões da Unidade de Terapia Intensiva Pediátrica e de prontuários de pacientes que estiveram internados em Unidade de Terapia Intensiva Pediátrica e fizeram uso de dispositivos invasivos entre janeiro de 2014 e dezembro de 2018. As análises estatísticas deste trabalho foram realizadas utilizando o software livre $R$ ( $R$ CORE TEAM 2019) e para testes de associação foi utilizado o teste U de Mann-Whitney. Resultados: Verificou-se que apenas os diagnósticos de neoplasias/ intoxicação/doenças de pele e sepse influenciaram no tempo de internação do paciente e o uso dos dispositivos: cateter venoso central, tubo orotraqueal, traqueóstomo, sonda nasogástrica, sonda orogástrica, sonda nasoenteral e cateter vesical de demora interferem no prolongamento do tempo de internação. Além disso, o número de pacientes infectados aumentou com o passar dos dias da hospitalização. Conclusão: O tempo de internação sofre influência do uso de dispositivos invasivos e tem associação com maior número de infecções.

\section{DESCRITORES}

Pediatria. Unidade de Terapia Intensiva Pediátrica. Tempo de Internação. Equipamentos e Provisões.

\begin{abstract}
Objective: To Investigate if the use of invasive devices leads to prolonged length of hospital stay in the Pediatric Intensive Care Unit of a University Hospital. Methodology: This was a quantitative, descriptive, cross-sectional and retrospective study, carried out at a University Hospital, where data from the Pediatric Intensive Care Unit admission book and medical records of patients who were hospitalized in the Pediatric Intensive Care Unit and used invasive devices were used, between January 2014 and December 2018. The statistical analyses were performed using the free software R (R CORE TEAM (2019)) and for association tests, the Mann-Whitney U test was used. Results: It was found that only the diagnoses of neoplasms/intoxication/skin diseases and sepsis influenced the patient's length of stay and the use of the devices: Central Venous Catheter, Orotracheal Tube, Tracheostome, Nasogastric Tube, Orogastric Tube, Nasoenteral Tube and Bladder catheter interferes in prolonging the length of hospital stay. In addition, the number of infected patients increased along the days of hospitalization. Conclusion: The length of hospital stay is influenced by using invasive devices and is associated with a greater number of infections.
\end{abstract}

\section{DESCRIPTORS}

Pediatrics. Pediatric Intensive Care Units. Length of Stay. Equipment and Supplies.

${ }^{1}$ Enfermeira. Pós-graduação pelo Programa de Residência Integrada Multiprofissional em Saúde Hospitalar da Universidade Federal da Paraíba-UFPB, Ênfase em Saúde da Criança e do Adolescente, João Pessoa-PB, Brasil.

${ }^{2}$ Enfermeira. Mestre em Enfermagem pela Universidade Federal de Pernambuco- UFPE. Enfermeira Assistencial do Hospital Universitário Lauro Wanderley-UFPB, João Pessoa-PB, Brasil.

${ }^{3}$ Enfermeira. Especialista em Terapia Intensiva pelo Programa de Residência da Secretaria Estadual de Saúde de Pernambuco. Especialista em Paciente Crítico pelo Hospital Sírio Libanês. Coordenadora da UTI Neonatal e Pediátrica do Hospital Universitário Lauro Wandeley-UFPB, João Pessoa-PB, Brasil.

${ }^{4}$ Enfermeira. Doutora em Enfermagem pela Universidade Federal da Paraíba- UFPB. Enfermeira da UTI Pediátrica do Hospital Universitário Lauro Wanderley- UFPB, João Pessoa- PB, Brasil. 
A s Unidades de Terapia Intensiva (UTIs) ainda são vistas pelo senso comum como lugares que recebem pacientes com pouca ou nenhuma expectativa de sobrevida devido à complexidade dos quadros de saúde. Porém, com terapias e atendimentos individualizados, aparatos médicos, cada vez mais tecnológicos e equipes interdisciplinares especializadas, tem-se conseguido resultados positivos, recuperação de saúde e aumento da sobrevida ${ }^{1}$.

Em pediatria, a hospitalização por si só é um processo traumático na vida da criança, sendo o hospital um ambiente estranho que causa uma quebra na rotina habitual, associado a outros fatores como o afastamento familiar, a ausência do ato de brincar e de outras situações estressoras decorrentes da internação, podendo produzir sentimentos como medo, insegurança e ansiedade, prejudiciais ao desenvolvimento da criança².

A internação em UTI Pediátrica traz diversas nuances para a criança e também para a família, causando alterações na dinâmica familiar. Ver o ente querido em um ambiente destinado a pacientes críticos ainda no início da vida, em uso de diversos equipamentos e dispositivos invasivos, sendo manipulado constantemente, desperta sentimentos de dúvida, dor e medo da morte, por não saber como esse evento irá finalizar ${ }^{3,4}$.

Com a realização de procedimentos complexos, o uso prolongado de antimicrobianos e de dispositivos invasivos, rotatividade de diversos especialistas, associado ao prolongamento do tempo de internação e por ser um ambiente altamente contaminado, a Unidade de Terapia Intensiva Pediátrica se configura como local propício ao surgimento de Infecções Relacionadas à Assistência a Saúde (IRAS) 5 uso de imunossupressores, período de internação prolongado, colonização por micro-organismos resistentes, prescrição de antimicrobianos e a própria característica do ambiente da UTI, além da condição clínica do paciente. O conhecimento do perfil bacteriano de cada cultura norteia a equipe médica no tratamento inicial das infecções. Objetivo: O objetivo desse trabalho foi verificar a ocorrência e o perfil bacteriano presente em pacientes internados na UTI de um hospital universitário. Material e métodos: $O$ estudo foi realizado através da análise de exames de secreções traqueais, hemoculturas e uroculturas de pacientes internados no período de janeiro a junho de 2018. Os dados foram coletados por meio de impressos laboratoriais do próprio serviço e tabulados na planilha do Excel ${ }^{\circledR}$, sendo divididos em amostras positivas e negativas, e realizada análise descritiva com valores absolutos e percentuais. Resultados: Em geral, as bactérias de maior ocorrência foram Acinetobacter baumannii $(20,3 \%$.

As IRAS são quaisquer infecções que acometem o indivíduo, durante sua permanência em instituições de saúde e que possam estar relacionadas a procedimentos, configurando-se como um grave problema de saúde pública, aumentando a morbimortalidade e os custos $^{5,6}$ uso de imunossupressores, período de internação prolongado, colonização por micro-organismos resistentes, prescrição de antimicrobianos e a própria característica do ambiente da UTI, além da condição clínica do paciente. $O$ conhecimento do perfil bacteriano de cada cultura norteia a equipe médica no tratamento inicial das infecções. Objetivo: O objetivo desse trabalho foi verificar a ocorrên- 
cia e o perfil bacteriano presente em pacientes internados na UTI de um hospital universitário. Material e métodos: O estudo foi realizado através da análise de exames de secreções traqueais, hemoculturas e uroculturas de pacientes internados no período de janeiro a junho de 2018. Os dados foram coletados por meio de impressos laboratoriais do próprio serviço e tabulados na planilha do Excel $\circledast$, sendo divididos em amostras positivas e negativas, e realizada análise descritiva com valores absolutos e percentuais. Resultados: Em geral, as bactérias de maior ocorrência foram Acinetobacter baumannii (20,3\%. Segundo Rodrigues e Pereira $(2016)^{6}$, no Brasil, aproximadamente, 5 a $15 \%$ dos pacientes hospitalizados e 25 a $35 \%$ dos pacientes admitidos em UTIs adquiram algum tipo de infecção relacionada à assistência à saúde, sendo ela a quarta causa de mortalidade.

Nas UTIs, os principais responsáveis pelas IRAS são os procedimentos invasivos como os cateterismos vesicais, os cateteres venosos centrais e a ventilação mecânica; além disso, o uso de antimicrobianos e de medicações imunossupressoras, o longo tempo de internação, a colonização por micro-organismos multirresistentes e o ambiente, são alguns dos fatores que favorecem as infecções ${ }^{7}$.

Nesse contexto, o enfermeiro é um importante agente no combate as IRAS por estar diretamente envolvido na prestação de cuidados ao paciente, identificando as necessidades destes para aplicação das intervenções necessárias ${ }^{7}$.

O prolongamento do tempo de internação em UTI, na maior parte dos casos, está relacionado à dificuldade de acesso a esses leitos, agravando os quadros de saúde dos pacientes que esperam por vaga, visto que o maior tempo de espera está ligado à piora clínica e a maiores índices de mortalidade, gerando diminuição da rotatividade e demandando maiores custos financeiros para unidade hospitalar ${ }^{8}$.

Logo, conhecer a interferência do uso de dispositivos invasivos no prolongamento do tempo de internação hospitalar é importante para que equipes profissional e gestora possam direcionar o atendimento ao paciente de forma que atenda suas necessidades e não aumente o tempo de ocupação do leito de UTI Pediátrica.

Diante do exposto, o presente estudo teve como objetivo averiguar se o uso de dispositivos invasivos leva ao prolongamento do tempo de internação na UTI Pediátrica de um Hospital Universitário.

\section{METODOLOGIA}

Tratou-se de um estudo quantitativo do tipo descritivo, transversal e retrospectivo, realizado na UTI Pediátrica de um Hospital Universitário Federal da Cidade de João Pessoa- PB, referência no estado para doenças raras e crônicas, que conta com 4 leitos de UTI Pediátrica, admitindo crianças a partir de 28 dias de vida até 14 anos, 11 meses e 29 dias, que estão em situações críticas, com doenças agudas e crônicas agudizadas e em pós-operatório.

O período de coleta deu-se de julho a dezembro de 2019, por meio de dados contidos em livro de admissões e alta e em prontuários de pacientes que ficaram internados de janeiro de 2014 a dezembro de 2018 na UTI, com amostra constituída por 110 prontuários. Todos os pacientes internados nesse período 
que fizeram uso de dispositivos invasivos foram incluídos, exceto aqueles que permaneceram na UTI menos de 24 horas, aqueles com diagnóstico de doenças crônicas degenerativas, que culminam com o prolongamento da internação na UTI e os que não tiveram o prontuário localizado no período da pesquisa.

A coleta de dados deu-se em dois momentos. Primeiramente, foi usado o Livro de Admissões do período estabelecido na pesquisa para obtenção de dados pessoais e números dos prontuários dos participantes. No segundo momento, os prontuários foram consultados no Serviço de Arquivo Médico e Estatística (SAME) e foi utilizado um instrumento de coleta que contava com 12 variáveis, com informações sociodemográficas e outras referentes à internação como: diagnóstico, tempo de internação, uso de dispositivos invasivos, tempo de permanência na unidade, diagnósticos de enfermagem e evolução do paciente.

Segundo registros no livro de admissões e altas do período, foram internados 219 pacientes no período citado, onde 110 compuseram a amostra. Foram eliminados 11 prontuários por estarem nos critérios de exclusão da pesquisa e 98 prontuários não foram localizados ou estavam com dados referentes à internação na UTI incompletos.

Após coletados, os dados foram transferidos para uma tabela para serem analisados. As análises estatísticas foram realizadas utilizando o software livre $R$ ( $R$ CORE TEAM (2019) e para testes de associação foi utilizado o teste $U$ de Mann-Whitney. Adotou-se $p<0,05$.

A pesquisa foi aprovada pelo Comitê de Ética em Pesquisa do Hospital Universitário Lauro Wanderley da Universidade Fede- ral da Paraíba- UFPB, através do CAAE $\mathrm{n}^{\circ}$ 15002619.8.0000.5183, em 12 de junho de 2019, respeitando-se a Resolução no 466 de 2012, do Conselho Nacional de Saúde no que diz respeito a pesquisa com seres humanos ${ }^{9}$.

\section{RESULTADOS}

De acordo com a análise, observou-se que dos 110 pacientes, $56 \%$ eram do sexo masculino e $44 \%$ do sexo feminino. Quanto à raça, $65 \%$ foram declarados pardos e a faixa etária predominante, $49 \%$, foi de lactentes (28 dias a 24 meses de idade). Ocorreram 4\% de internações de neonatos e $1 \%$ de internação de adolescente, maior de 15 anos, possivelmente, por falta de vagas nas UTIs neonatal e adulto.

No que tange a procedência, $54,6 \%$ dos pacientes que se internaram foram encaminhados de outros setores do Hospital Universitário, $19,1 \%$ de Hospitais de João Pessoa e da Região Metropolitana, que engloba os municípios de Cabedelo, Bayeux, Santa Rita, Conde, Lucena, Mamanguape, Rio Tinto e Cruz do Espírito Santo; 13,6\% de hospitais das demais regiões do estado; $11,8 \%$ das Unidades de Pronto Atendimento (UPA) e $0,9 \%$ de hospital particular.

Em decorrência da diversidade de diagnósticos encontrados, estes foram agrupados por sistemas, para melhor entendimento, sendo considerados até três diagnósticos por paciente. As doenças do sistema respiratório (insuficiência respiratória aguda, pneumonia, asma, derrame pleural, empiema, atelectasia, estenose subglótica, bronquiolite obliterante, broncodisplasia, hemangioma de rino e orofaringe, sinusopatia, hipertensão pulmonar, encurtamento de pregas ariepiglóticas, 
laringomalácia e fibrose cística) tiveram maior prevalência, aparecendo em $46 \%$ dos casos, acompanhado de pós-operatórios, doenças do sistema urinário (infecção do trato urinário, insuficiência renal aguda, hipertensão arterial, desequilíbrio hidreoletrolítico, injúria renal aguda, hifronefrose, nefrite lúpica) e sepse, com $21 \%, 17 \%$ e $15 \%$ dos casos, respectivamente.
Ao analisar a Tabela 1, observa-se o comportamento dos diagnósticos em relação ao tempo de internação, percebendo-se que com o passar dos dias cresce a quantidade de pacientes diagnosticados com doenças do sistema imunológico e sepse.

$\mathrm{Na}$ Tabela 1, verifica-se ainda que apenas os diagnósticos de neoplasias/ intoxicação/doenças de pele e sepse tiveram

Tabela 1. Tipo de diagnóstico por faixa de dias de internação e associação dos tipos de diagnósticos com o tempo de internação dos pacientes em estudo.

\begin{tabular}{|c|c|c|c|c|c|c|c|c|}
\hline \multirow[t]{2}{*}{ Diagnósticos } & & \multicolumn{5}{|c|}{$\begin{array}{l}\text { Internação } \\
\text { (Dias) }\end{array}$} & \multirow[t]{2}{*}{$\begin{array}{c}\text { Total } \\
n=110\end{array}$} & \multirow[t]{2}{*}{ Valor $p^{*}$} \\
\hline & & 1 & 2 & 3 a 4 & 5 a 7 & $\geq 8$ & & \\
\hline \multirow{2}{*}{$\begin{array}{l}\text { Doenças do Sist. } \\
\text { Cardiovascular }\end{array}$} & Sim & 03 & 01 & 03 & 01 & 04 & 12 & \multirow[t]{2}{*}{0,717} \\
\hline & Não & 19 & 17 & 21 & 21 & 20 & 98 & \\
\hline \multirow{2}{*}{$\begin{array}{l}\text { Doenças do Sist. } \\
\text { Respiratório }\end{array}$} & Sim & 09 & 06 & 13 & 09 & 14 & 51 & \multirow[t]{2}{*}{0,194} \\
\hline & Não & 13 & 12 & 11 & 13 & 10 & 59 & \\
\hline \multirow{2}{*}{$\begin{array}{l}\text { Doenças do Sist. } \\
\text { Digestório }\end{array}$} & Sim & 3 & - & 02 & 03 & 05 & 13 & \multirow[t]{2}{*}{0,170} \\
\hline & Não & 19 & 18 & 22 & 19 & 19 & 97 & \\
\hline \multirow{2}{*}{$\begin{array}{c}\text { Doenças do Sist. } \\
\text { Urinário }\end{array}$} & Sim & 05 & 03 & 05 & 03 & 03 & 19 & \multirow[t]{2}{*}{0,451} \\
\hline & Não & 17 & 15 & 19 & 19 & 21 & 91 & \\
\hline \multirow{2}{*}{$\begin{array}{c}\text { Doenças do Sist. } \\
\text { Endócrino }\end{array}$} & Sim & - & 02 & - & - & 01 & 3 & \multirow[t]{2}{*}{0,832} \\
\hline & Não & 22 & 16 & 24 & 22 & 23 & 107 & \\
\hline \multirow{2}{*}{$\begin{array}{c}\text { Doenças do Sist. } \\
\text { Nervoso }\end{array}$} & Sim & 01 & 03 & 03 & 02 & 02 & 11 & \multirow[t]{2}{*}{0,833} \\
\hline & Não & 21 & 15 & 21 & 20 & 22 & 99 & \\
\hline \multirow{2}{*}{$\begin{array}{l}\text { Doenças do Sist. } \\
\text { Imunológico }\end{array}$} & Sim & 01 & 01 & 01 & 03 & 03 & 09 & \multirow[t]{2}{*}{0,156} \\
\hline & Não & 21 & 17 & 23 & 19 & 21 & 101 & \\
\hline \multirow{2}{*}{$\begin{array}{c}\text { Doenças do Sist. } \\
\text { Hematológico }\end{array}$} & Sim & 01 & - & 03 & - & 03 & 07 & \multirow[t]{2}{*}{0,191} \\
\hline & Não & 21 & 18 & 21 & 22 & 21 & 103 & \\
\hline \multirow{3}{*}{$\begin{array}{c}\text { Malformações } \\
\text { Congênitas e } \\
\text { Síndromes Genéticas }\end{array}$} & & & & & & & & \multirow{3}{*}{0,382} \\
\hline & Sim & 05 & 02 & 02 & 03 & 03 & 15 & \\
\hline & Não & 17 & 16 & 22 & 19 & 21 & 95 & \\
\hline \multirow{2}{*}{ Pós Operatório } & Sim & 04 & 03 & 05 & 08 & 03 & 23 & \multirow[t]{2}{*}{0,795} \\
\hline & Não & 18 & 15 & 19 & 14 & 21 & 87 & \\
\hline \multirow{2}{*}{$\begin{array}{c}\text { Neoplasias/ } \\
\text { Intoxicação/ Doenças } \\
\text { de pele }\end{array}$} & Sim & 03 & 03 & 03 & 01 & - & 10 & \multirow[t]{2}{*}{0,030} \\
\hline & Não & 19 & 15 & 21 & 21 & 24 & 100 & \\
\hline \multirow{2}{*}{ Sepse } & Sim & 02 & - & 03 & 05 & 06 & 16 & \multirow[t]{2}{*}{0,010} \\
\hline & Não & 20 & 18 & 21 & 17 & 18 & 94 & \\
\hline
\end{tabular}

${ }^{*} p<0,05$ 
associação significativa com o tempo de internação, apresentando valores de $p$ iguais a 0,03 e 0,01, respectivamente. Logo, esses diagnósticos influenciam no tempo de internação do paciente, sendo que para as neoplasias/intoxicação/doenças de pele o número de pacientes internados diminui com o tempo e, para sepse, esses números aumentam.

$\mathrm{Na}$ Tabela 2, em relação ao uso de dispositivos, percebe-se que à medida que o tempo de internação aumenta, a quantidade de pacientes que usaram cateter venoso central, tubo orotraqueal, traqueóstomo, sonda nasogástrica, sonda orogástrica, sonda na- soenteral e cateter vesical de demora também aumenta. Portanto, o uso destes dispositivos é mais frequente para tempo de internação mais longo. Ressaltando-se que o paciente pode ter feito uso de mais de um dispositivo invasivo.

Ainda na Tabela 2, verifica-se que o uso dos dispositivos: cateter venoso central $(p<0,001)$, tubo orotraqueal $(p<0,001)$, traqueóstomo $(p=0,025)$, sonda nasogástrica $(p<0,001)$, sonda orogástrica $(p=0,002)$, sonda nasoenteral $(p=0,001)$ e cateter vesical de demora $(p=0,009)$ tiveram uma associação positiva com o tempo de internação. Portanto, o uso de dispositivos invasivos interfere

Tabela 2. Uso de dispositivo por faixa de dias de internação do paciente e associação entre o uso de dispositivo com o tempo de internação dos pacientes em estudo.

\begin{tabular}{|c|c|c|c|c|c|c|c|c|}
\hline \multirow{2}{*}{\multicolumn{2}{|c|}{ Dispositivos }} & \multicolumn{5}{|c|}{$\begin{array}{c}\text { Internação } \\
\text { (Dias) }\end{array}$} & \multirow{2}{*}{$\begin{array}{c}\text { Total } \\
(n=110)\end{array}$} & \multirow[b]{2}{*}{ Valor $p^{*}$} \\
\hline & & 1 & 2 & 3 a 4 & 5 a 7 & $\geq 8$ & & \\
\hline Cateter Venoso & Sim & 20 & 17 & 15 & 14 & 19 & 85 & 0,074 \\
\hline Periférico & Não & 02 & 01 & 09 & 08 & 05 & 25 & \\
\hline \multirow{2}{*}{ Cateter Venoso Central } & $\operatorname{Sim}$ & 02 & 02 & 10 & 16 & 22 & 52 & $<0,001$ \\
\hline & Não & 20 & 16 & 14 & 06 & 02 & 58 & \\
\hline \multirow{2}{*}{ Tubo Orotraqueal } & Sim & 01 & - & 07 & 06 & 16 & 30 & $<0,001$ \\
\hline & Não & 21 & 18 & 17 & 16 & 08 & 80 & \\
\hline \multirow{2}{*}{ Traqueóstomo } & Sim & - & 01 & - & 02 & 03 & 06 & 0,025 \\
\hline & Não & 22 & 17 & 24 & 20 & 21 & 104 & \\
\hline \multirow{2}{*}{ Sonda Nasogástrica } & Sim & 02 & 02 & 09 & 09 & 17 & 39 & $<0,001$ \\
\hline & Não & 20 & 16 & 15 & 13 & 07 & 71 & \\
\hline \multirow{2}{*}{ Sonda Orogástrica } & $\operatorname{Sim}$ & 02 & 01 & 04 & 10 & 08 & 25 & 0,002 \\
\hline & Não & 20 & 17 & 20 & 12 & 16 & 85 & \\
\hline \multirow{2}{*}{ Sonda Nasoenteral } & Sim & - & - & 01 & 03 & 05 & 09 & 0,001 \\
\hline & Não & 22 & 18 & 23 & 19 & 19 & 101 & \\
\hline \multirow{2}{*}{$\begin{array}{l}\text { Cateter Vesical de } \\
\text { Demora }\end{array}$} & $\operatorname{Sim}$ & 02 & 03 & 06 & 08 & 09 & 28 & 0,009 \\
\hline & Não & 20 & 15 & 18 & 14 & 15 & 82 & \\
\hline \multirow{2}{*}{ Drenos } & Sim & 03 & 01 & 03 & 02 & 06 & 15 & 0,404 \\
\hline & Não & 19 & 17 & 21 & 20 & 18 & 95 & \\
\hline \multirow{2}{*}{ Outros } & Sim & 03 & 02 & 06 & 02 & 05 & 18 & 0,397 \\
\hline & Não & 19 & 16 & 18 & 20 & 19 & 92 & \\
\hline
\end{tabular}

${ }^{*} p<0,05$ 
diretamente no prolongamento do tempo de internação em UTI Pediátrica.

Ao analisar a Tabela 3, observa-se que o tempo médio de uso dos dispositivos invasivos assume valores pequenos devido ao grande número de pacientes que não usaram determinado dispositivo. Os dispositivos cateter venoso central e sonda nasogástrica apresentaram maior variabilidade, apresentando desvios padrões de 8,07 e 9,5, respectivamente. Estes dispositivos variaram entre 0 - 58 dias de internação para cateter venoso central e 0 - 76 dias em relação à sonda nasogástrica.

Também avaliou-se a associação entre infecções adquiridas durante a internação e o tempo de internação. Como representado na Figura 1, o número de pacientes infectados aumentou com o passar dos dias da hospitalização. Até quatro dias de internação não houve casos, começando a surgir a partir do quinto dia, sendo observado maior número de infecções a partir de 8 dias de internação. $O$ teste de associação apresentou valor $p$
$<0,001$, considerando-se uma associação significativa entre a infecção e o tempo de internação.

Em relação aos diagnósticos de enfermagem, os mais presentes foram risco de infecção, integridade da pele/tissular prejudicada e padrão respiratório ineficaz, com $84 \%$, $78 \%$ e $59 \%$, respectivamente.

Quanto à evolução, $82 \%$ dos pacientes receberam alta para enfermaria, $8 \%$ foram a óbito, $6 \%$ foram transferidos para outros hospitais e $4 \%$ foram transferidos para outra UTI no próprio hospital.

\section{DISCUSSÃO}

Por ser um ambiente altamente contaminado a UTI Pediátrica é um importante local de risco para IRAS. Alguns fatores podem ser predisponentes ao desenvolvimento de infecções, como pacientes com idade inferior a 2 anos, portadores de imunodeficiências e doenças de base graves. Também são determinantes os dias de internação na UTI e os

Tabela 3. Valores de média, mediana, desvio padrão, mínimo e máximo para o tempo de uso dos dispositivos e tempo de internação.

\begin{tabular}{lccccc}
\hline \multicolumn{1}{c}{ Variáveis } & Média & Mediana & Desvio Padrão & Mínimo & Máximo \\
\hline Cateter Venoso Periférico & 2,21 & 1 & 3,59 & - & 32 \\
Cateter Venoso Central & 4,36 & - & 8,07 & - & 58 \\
Tubo Orotraqueal & 1,69 & - & 4,28 & - & 25 \\
Traqueóstomo & 1,16 & - & 7,43 & - & 66 \\
Sonda Nasogástrica & 3,36 & - & 9,5 & - & 76 \\
Sonda Orogástrica & 1,42 & - & 4,4 & - & 30 \\
Sonda Nasoenteral & 0,99 & - & 5,37 & - & 51 \\
Cateter Vesical de Demora & 1,40 & - & 3,83 & - & 25 \\
Drenos & 0,80 & - & 2,9 & - & 23 \\
Outros* & 0,81 & - & 2,69 & - & 20 \\
Tempo de Internação & 6,83 & 4 & 10,3 & 1 & 76 \\
\hline "Cateter central de inserção periférica (picc), gastrostomia, cateterismo vesical intermitente, \\
port-a-cath, derivação ventrículo peritoneal, sonda replogue. & &
\end{tabular}


Figura 1. Casos de infecção por dias de internação

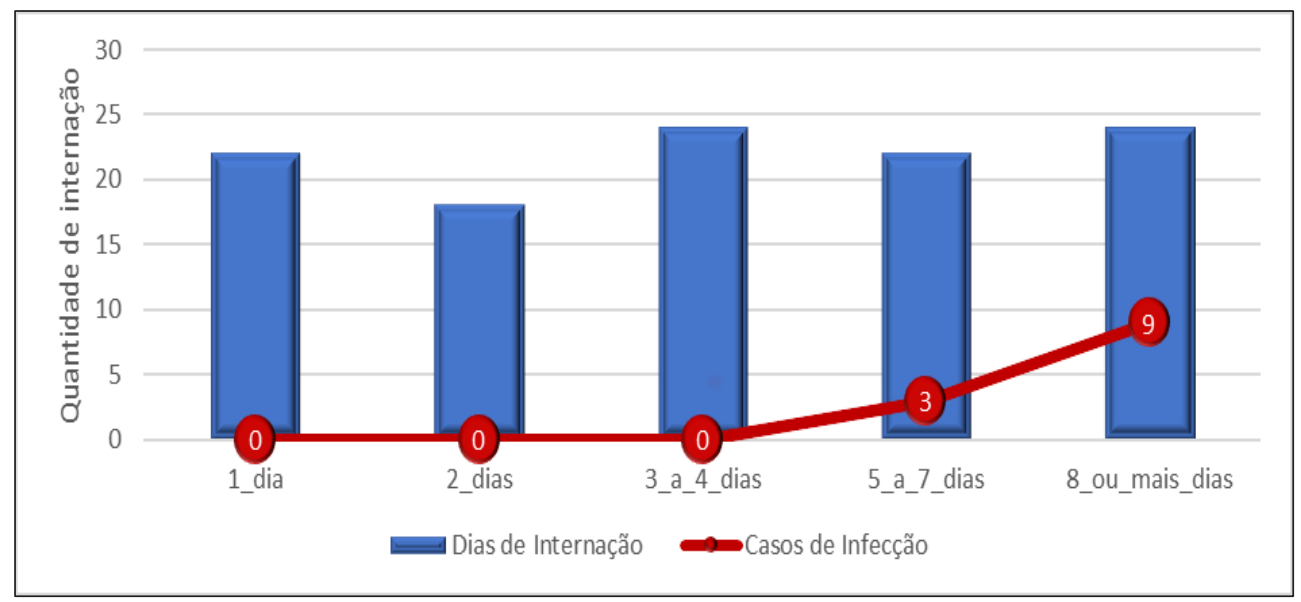

dias de uso de dispositivos invasivos, como cateteres venosos centrais e ventilação mecânica ${ }^{10}$.

A ocorrência de internações em UTI Pediátrica é prevalente no sexo masculino quando comparado ao sexo feminino ${ }^{11,12}$, confirmando o achado deste estudo, onde $56 \%$ dos pacientes eram do sexo masculino.

A faixa etária de maior admissão foi de lactentes, equivalente ao estudo realizado na Bolívia. Pode-se associar o maior número de lactentes a diversos fatores que levam ao adoecimento em crianças nesse intervalo de idade, como a fragilidade imunológica, as baixas taxas de aleitamento materno, o esquema vacinal incompleto e os déficits nutricionais ${ }^{10}$ que podem ocasionar a necessidade de cuidados intensivos.

A causa mais frequente de internação em UTI Pediátrica, segundo Batista et $\mathrm{al}^{13}$, são as doenças do sistema respiratório, como: insuficiência respiratória aguda, pneumonia, derrame pleural e asma, corroborando com os resultados de outro estudo realizado em UTIP de um hospital infantil da Cidade de João Pessoa-PB, onde $47,05 \%$ dos pacientes foram internados por patologias pulmonares ${ }^{14}$. Deve-se levar em consideração que pneumonias correspondem a grande parte das afecções respiratórias, sendo as infecções mais comuns em UTIs ${ }^{6}$, atestando o que foi encontrado neste estudo, onde $46 \%$ dos pacientes foram acometidos por afecções respiratórias.

O número considerável de internações de pacientes em pós-operatório na UTI, $21 \%$ das admissões, deve-se a referência do hospital para cirurgias pediátricas no estado e a necessidade de cuidados intensivos após determinados procedimentos cirúrgicos, o que impede o acompanhamento do pós-operatório imediato na enfermaria. Mesmo não contando com serviço de emergência e realizando procedimentos cirúrgicos de forma eletiva, o número de admissões foi próximo ao obtido por outros autores, onde o hospital estudado oferecia atendimento de emergência ${ }^{13}$.

Infecções do trato urinário quando não devidamente tratadas, podem resultar em alterações funcionais e morfológicas dos rins e das vias urinárias, causando em longo prazo outras afecções como pressão alta e insuficiência renal. $O$ tratamento precoce 
evita que complicações aconteçam, porém, em crianças, os sintomas podem ser inespecíficos levando ao agravamento do quadro, o que pode ter ocasionado o número considerável de internações por doenças do sistema urinário ${ }^{13,15}$, que foram responsáveis por $17 \%$ das internações.

O diagnóstico de sepse esteve presente em $15 \%$ das internações. Por ser causada por diversos patógenos e ter diferentes formas de manifestação, acaba tornando-se de difícil diagnóstico, retardando o tratamento. Pode ter origem em mais de um sítio do organismo, onde infecções respiratórias se apresentam como sítio de infecção mais comum de sep$\mathrm{se}^{16}$. Estudo realizado em UTI Pediátrica de Campinas-SP obteve taxas de internação por sepse de $14,9 \%$, número semelhante ao encontrado neste estudo ${ }^{17}$.

Observou-se ainda que pacientes diagnosticados com sepse contaram com tempo mais longo de internação, ratificando um estudo multicêntrico realizado em UTI Pediátrica da América Latina que mostra que o tempo de internação foi maior nos pacientes com sepse quando comparado a outros diagnósticos ( $p<0,001)$, demandando de maior número de intervenções e maiores custos para o hospital, além do uso de drogas vasoativas ${ }^{16}$.

Pacientes diagnosticados com neoplasias, intoxicação e doenças de pele apresentaram menor tempo de permanência em UTI Pediátrica. Deve-se considerar que os diagnosticados com neoplasias na UTIP do estudo são regulados para hospitais especializados e transferidos assim que possível. Assim, o número de admissões e tempo de permanência desses pacientes foi inferior ao encontrado em estudo realizado em UTI Pediátrica no Estado de Pernambuco, onde neoplasias foram a principal causa de internamentos ${ }^{18}$. Pacientes internados por intoxicação são transferidos para enfermaria assim que o quadro clínico é estabilizado. Aqueles com doenças de pele podem ter maiores complicações devido à perda da proteção epidérmica, estando mais suscetíveis à infecções, resultando em sepse e óbito precoce ${ }^{17}$, com poucos dias de internação.

O uso de dispositivos invasivos é uma prática comum e necessária em UTI, no entanto, devido à fragilidade do sistema imunológico do paciente e fatores intrínsecos de cada caso, existe maior chance de desenvolvimento de infecções associadas aos dispositivos, o que pode representar um fator importante no prolongamento de internações ${ }^{19}$.

Pesquisas mostram que de $70 \%$ a $80 \%$ dos pacientes internados fazem uso de algum dispositivo intravascular. Os cateteres venosos periféricos são os dispositivos mais utilizados em unidades hospitalares, cerca de 60 vezes mais usados que o cateter venoso central. As taxas de infecção são menores quando comparadas a de cateteres centrais, tendo em vista que sendo observado os sinais de flebite é possível a troca imediata do cateter ${ }^{20,21}$. O tempo médio de permanência de cateter venoso periférico encontrado neste estudo foi de 2,21 dias, dado consoante com a literatura, em que a média é de 2 dias em pediatria ${ }^{22}$.

No entanto, o cateter venoso central é a escolha mais frequente para pacientes que necessitam de longos períodos de terapia intravenosa e com rede venosa periférica de difícil acesso ${ }^{7}$. Este tipo de cateter é uma importante porta de entrada para infecções, onde são relatadas na literatura altas taxas de bacteremia associadas ao uso do cateter 
venoso central, relacionando ao maior tempo de internação ${ }^{20}$, o que confirma dado encontrado $(P<0,001)$. Estudo realizado em UTI Pediátrica da Turquia traz o tempo médio de permanência de cateter central de 10,58 dias $^{23}$, número superior ao encontrado neste estudo (4,36 dias).

Além dos dispositivos intravasculares, paciente em UTI tem maior probabilidade de usar ventilação mecânica devido à elevada chance de apresentar problemas respiratórios. Em uma UTI Pediátrica no Ceará, o tempo médio de uso de ventilação mecânica invasiva foi superior há 8 dias, ficando evidenciado que nos meses em que a ventilação mecânica invasiva foi mais usada o tempo de internação foi prolongado ${ }^{12}$, dado similar ao encontrado no estudo, em que se observa que o uso de tubo orotraqueal e o traqueóstomo foi crescente com o passar dias, com maior número de pacientes que usaram 8 dias ou mais.

O paciente em uso de ventilação mecânica e tubo orotraqueal por tempo prolongado pode vir a desenvolver pneumonia associada a ventilação (PAV), principal infecção relacionada a assistência à saúde, responsável por longos períodos de internação e altos índices de mortalidade ${ }^{7}$. Neste estudo, 16 pacientes usaram o tubo orotraqueal por período de 8 ou mais dias, fator que pode facilitar desenvolvimento da PAV e levar a indicação de traqueostomia.

A indicação de traqueostomia é respaldada pelo longo tempo de intubação e ventilação, além de anormalidades de vias aéreas superiores em que esse procedimento é recomendado ${ }^{24}$. Pode-se perceber que o número de pacientes traqueostomizados também se eleva com o passar dos dias, inferindo-se que além daqueles em que a traqueostomia foi indicada por anormalidades anatômicas, aqueles com muitos dias de intubação orotraqueal tiveram o procedimento indicado.

Sondas de alimentação também são dispositivos muito usados em pediatria quando a alimentação oral não é possível. Cerca de $25 \%$ de crianças internadas precisam fazer uso de sondas de alimentação até a estabilização clínica, onde a mais usada é a sonda nasogástrica ${ }^{25}$. A sonda de alimentação mais utilizada pelos pacientes deste estudo foi a sonda nasogástrica (39 pacientes), apresentando maior tempo médio de uso entre os outros tipos de sonda de alimentação (3,36 dias).

O cateter vesical de demora é um dos dispositivos mais usados nas unidades de saúde e também um grande responsável por infecções do trato urinário. A contaminação do sistema do cateter pode ocorrer no momento da inserção, durante o manuseio diário ou devido ao longo período de uso. Em dados colhidos em duas UTI do Rio de Janeiro, observou-se que o tempo médio de permanência do cateter foi de 8 a 15 dias, número superior ao encontrado neste estudo (1,4 dias) e, quanto maior o tempo de uso, maiores as chances de desenvolver infecção do trato urinário, elevando-se os custos de internação e a necessidade de leitos de $\mathrm{UTI}^{7,26}$. De acordo com os resultados encontrados, o número de pacientes que necessitaram usar o cateter foi crescente com o passar do tempo, fator que favorece o surgimento de IRAS.

As IRAS são classificadas como qualquer infecção adquirida durante a internação hospitalar. Para fins de classificação, aquelas infecções que se apresentam após 72 horas de internação ou as que se manifestam antes desse período e são relacionadas a procedimentos realizados no período da internação 
também são classificadas IRAS ${ }^{7}$. Neste estudo, as infecções adquiridas na internação foram registradas a partir do quinto dia de hospitalização em UTI, existindo crescimento no número de casos com o passar do tempo, entendendo-se que quanto maior o tempo de internação, maior o número de pacientes infectados.

Os profissionais de enfermagem são fundamentais para os cuidados prestados em UTI. Os diagnósticos de enfermagem se configuram como uma etapa da sistematização da assistência, sendo fundamental sua identificação para correta implementação do cuidado, de forma que o atendimento prestado pela equipe de enfermagem seja personalizado e atenda às necessidades do paciente e da família. Os diagnósticos de enfermagem mais prevalentes neste estudo foram risco de infecção, integridade da pele/tissular prejudicada e padrão respiratório ineficaz, diagnósticos semelhantes aos obtidos em uma UTIP de Campina Grande-PB ${ }^{27}$.

O número de óbitos ( $8 \%$ ) foi menor em relação a outros relatos na literatura ${ }^{13,17}$, podendo ser justificado pela diferença do público atendido pelos hospitais.

Os dados obtidos através desse trabalho permitiram entender o perfil sociodemográfico dos pacientes atendidos na UTIP, bem como analisar a relação de alguns diagnósticos e dispositivos invasivos como fator determinante no prolongamento do tempo de internação.

\section{REFERÊNCIAS}

1. Silveira NR, Nascimento ERP do, Rosa LM da, Jung W, Martins SR, Fontes MDS. Palliative care and the intensive care nurses: feelings that endure. Rev Bras Enferm. 2016; 69(6):1074-81.
No entanto este estudo apresentou limitações por se tratar de estudo retrospectivo com dados obtidos em prontuários, onde um número considerável não foi localizado, incluindo grande parte dos óbitos ocorridos no período e, alguns, continham informações incompletas sobre o período de internação na UTI.

\section{CONCLUSÃO}

Os dispositivos invasivos são importantes aliados no sucesso de tratamentos, porém o tempo de uso é fator determinante no tempo de internação do paciente.

Conclui-se que pacientes que fizeram uso de dispositivos invasivos tiveram o tempo de internação em UTI Pediátrica prolongado, especialmente aqueles que fizeram uso de cateter venoso central, tubo orotraqueal, traqueóstomo, sonda nasogástrica, sonda orogástrica, sonda nasoenteral e cateter vesical de demora.

Assim, aumenta-se os dias de ocupação de leitos de UTI, onde no Brasil é um setor em que a procura é maior que a demanda. Além disso, com o longo tempo de internação, o paciente se torna mais suscetível ao desenvolvimento de infecções.

Logo, se faz necessário a sensibilização dos profissionais para importância do uso racional dos dispositivos invasivos. Devendo-se analisar o caso e a necessidade, para que as internações não se prolonguem devido ao uso excessivo. 
3. Sousa AS, Cabeça LPF, Souza MA, Melo L de L. Experiências maternas diante da transferência do filho para a unidade de terapia intensiva. Rev Baiana Enferm. 2018;32:1-9.

4. Ferreira MJDM, Dodt RCM, Lima AM, Marques DR de F, Pinheiro SMPR. Percepção Dos Acompanhantes Sobre Dispositivos Invasivos Em Uma Unidade De Terapia Intensiva Pediátrica. Enferm Foco. 2018; 9(2):18-22.

5. Naue CR, Ribeiro T, Ribeiro R, Batista K, Aquino S. Ocorrência e perfil bacteriano de culturas coletadas em pacientes internados na unidade de terapia intensiva em um hospital terciário. HU Rev. 2019;45(2):122-133.

6. Rodrigues CN, Pereira DCA. Infecções relacionadas à assistência à saúde ocorridas em uma Unidade de Terapia Intensiva. Rev Investig Bioméd. 2016; 8:41-51.

7. Araújo PL, Mendonça AE oliveira de, Medeiros RÁ de, Souza Neto VL, Nobre TTX, Costa IKF. Prevalência de infecção relacionada à assistência à saúde em pacientes internados em unidade de terapia intensiva. Enferm Glob. 2018;17(52):291-303.

8. Goldwasser RS, Lobo SMDC, Arruda EF, Angelo SA, Lapa e Silva JR, Salles AA, et al. Dificuldades de acesso e estimativas de leitos públicos para unidades de terapia intensiva no estado do Rio de Janeiro. Rev Saúde Pública. 2016;1-10.

9. Brasil, Ministério da Saúde. Resolução n 466, de 12 de dezembro de 2012. Diretrizes e normas regulamentadoras de pesquisa em seres humanos. Diário Oficial da União, Brasília. 2012;

10. Lesczinsky MC, Abularach ZV. Gérmenes más frecuentes en infecciones asociadas a la atentíon en salud en la UTI Pediátrica. Rev Cient Cienc Méd. 2017; 20(1):20-25.

11. Licorish MP, Castellanos MCM, Soto ZM, Amaro AC, Founier EL. Caracterización clínica y microbiológica de niños y adolescentes con infecciones asociadas a la atención sanitaria. MEDISAN. 2018;22(5):508-17.

12. Oliveira CAS de, Pinto FCC, Vasconcelos TB de, Bastos VPD. Análise de indicadores assistenciais em uma Unidade de Terapia Intensiva Pediátrica na cidade de Fortaleza/CE. Cad Saúde Colet. 2017; 25(1):99-105

13. Onofre BWN, Coelho MC de R, Trugilho SM, Pinasco GC, Santos FE de S, Ramos-silva V. Perfil clínico-epidemiológico de pacientes internados em unidades de cuidados intensivos pediátricos. J Hum Growth Dev. 2015; 25(2):187-93.

14. Muniz ADL de O, Dias ML da C. Perfil clínico-epidemiológico de pacientes com Síndrome do Desconforto Respiratório Agudo em UTI pediátrica. Vol. 11, Revista Cereus. 2019.

15. Troche AV, Ortiz-cuquejo LM, Samudio-dominguez GC, Mauro A, González C, Lascurain A. Prevalencia de uropatógenos y sensibilidad antimicrobiana en lactantes menores de 2 años provenientes de la comunidad con diagnóstico de infección de vías urinarias Prevalence of uropathogens and antimicrobial susceptibility in infants younger than 2 year. Rev Nac. 2016; 8(2):34-46.

16. Souza DC de. Epidemiologia da sepse em crianças internadas em unidades de terapia intensiva pediátrica da América
Latina. (Tese Doutorado) São Paulo Fac Med da Univ São Paulo. 2016;155p.

17. Pedro T da CS, Morcillo AM, Baracat ECE. Etiologia e fatores prognósticos da sepse em crianças e adolescentes admitidos em terapia intensiva. Rev Bras Ter Intensiva. 2015; 27(3):240-6.

18. Mendonça JG de, Guimarães MJB, Mendonça VG de, Portugal JL, Mendonça CG de. Perfil das internações em Unidades de Terapia Intensiva Pediátrica do Sistema Único de Saúde no estado de Pernambuco, Brasil. Ciênc Saúde Colet. 2019; 24(3):907-16.

19. Arias AV, Garcell HG, Amis A, Cardoso H, Corporation HM Infección asociada a dispositivos en Unidad de Cuidados Intensivos. Oeste de Qatar. Rev Haban Cienc Méd. 2019;18(2):231-40.

20. Ruiz-giardin JM, Chamorro IO, Ríos LV, Aroca JJ, Arata MIG, López JVS, et al. Blood stream infections associated with central and peripheral venous catheters. BMC Infect Dis. 2019;19(841):1-9.

21. Høvik LH, Gjeilo KH, Lydersen S, Rickard CM, Røtvold B, Damås JK, et al. Monitoring quality of care for peripheral intravenous catheters; feasibility and reliability of the peripheral intravenous catheters mini questionnaire ( PIVC-miniQ ). BMC Infect Dis. 2019;19(636):1-10.

22. Oliveira J De, Miranda F, Castelo R, Santana B De, Camargo CL De, Lopes C, et al. Acessos Venosos Periféricos em crianças hospitalizadas: um estudo fotográfico. Rev Enferm UFPE line. 2016;10(2):701-7.

23. Tolunay İ, Yıldızdaş RD, Elçi H, Alabaz D. Assessment of central venous catheterization and complications in a tertiary pediatric intensive care unit. Turkish $\mathrm{J}$ Pediatr. 2018;60(1):63-9.

24. Siegert $O A$, Jaramillo G C, Gonzáles $C A D$, Alba P R, Azcárate $Y$ T. Indicaciones, hallazgos broncoscópicos y mortalidad en pacientes pediátricos con traqueostomía en un hospital de México. Neumol Pediatr. 2017;12(2):85-89.

25. Lyman B, Kemper C, Northington L, Yaworski JA, Wilder K, Moore C, et al. Use of Temporary Enteral Access Devices in Hospitalized Neonatal and Pediatric Patients in the United States. JPEN J Parenter Enteral Nutr. 2016; 40(4):574-580.

26. Tolentino ACDMS, Peregrino AAF, Marta CB, Silva RCL, Schutz V. O custo do cateterismo vesical de demora nos pacientes internados na Unidade de Terapia Intensiva. J Res Fundam Care. 2017; 9(4):1170-6.

27. Guedes DMB, Rossato LM, Oliveira EA. Diagnósticos de enfermagem mais frequentes em uma unidade de terapia intensiva pediátrica. Rev Enferm UFSM. 2015;5(3):476-85.

\section{CORRESPONDÊNCIA}

Maria Mayara Araújo Pereira

Rua Doutor Ricardo Tavares de Melo, 10

Loteamento Arthur Tavares

Ferreiros-PE, 55880-000

E-mail: mmayaraaraujo@gmail.com 